\title{
一 データ・チヤート
}

\section{應 力 換 算 表}

Tons per sq. in. to psi.

$\mathrm{Kg}$. per sq. $\mathrm{mm}$. to psi.

\begin{tabular}{|c|c|c|c|c|c|c|c|c|c|c|c|}
\hline $\begin{array}{l}\text { Tons Per } \\
\text { sq. in. }\end{array}$ & psi. & {$\left[\begin{array}{l}\text { Tons per } \\
\text { sq. in. }\end{array}\right.$} & psi. & $\begin{array}{l}\text { Tons per } \\
\text { sq. in. }\end{array}$ & psi. & $\begin{array}{r}\mathrm{Kg} . \text { per } \\
\text { sq. } \mathrm{Mm} .\end{array}$ & psi. & {$\left[\begin{array}{c}\mathrm{Kg} \cdot \mathrm{per} \\
\mathrm{sg} \cdot \mathrm{Mm} .\end{array}\right.$} & psi. & $\begin{array}{l}\mathrm{Kg} \cdot \mathrm{per} \\
\mathrm{sq} \cdot \mathrm{mm} \text {. }\end{array}$ & psi. \\
\hline $\begin{array}{l}10.0 \\
10.5 \\
11.0 \\
11.5 \\
12.0 \\
12.5 \\
13.0 \\
13.5 \\
14.0 \\
14.5\end{array}$ & $\begin{array}{l}22,400 \\
23,520 \\
24,640 \\
25,770 \\
26,880 \\
28,000 \\
29,120 \\
30,240 \\
31,360 \\
32,480\end{array}$ & $\begin{array}{l}35.0 \\
35.5 \\
36.0 \\
36.5 \\
37.0 \\
37.5 \\
38.0 \\
38.5 \\
38.0 \\
39.5\end{array}$ & $\begin{array}{l}78,400 \\
79,520 \\
80,640 \\
81,760 \\
82,880 \\
84,000 \\
85,120 \\
86,240 \\
87,360 \\
88,480\end{array}$ & $\begin{array}{l}70 \\
71 \\
72 \\
73 \\
74 \\
75 \\
76 \\
77 \\
78 \\
79\end{array}$ & $\begin{array}{l}156,800 \\
159,040 \\
161,280 \\
163,520 \\
165,760 \\
168,000 \\
170,240 \\
172,480 \\
174,720 \\
176,960\end{array}$ & $\begin{array}{l}10 \\
11 \\
12 \\
13 \\
14 \\
15 \\
16 \\
17 \\
18 \\
19\end{array}$ & $\begin{array}{l}14,223 \\
15,646 \\
17,068 \\
18,490 \\
18,913 \\
21,335 \\
22,757 \\
24,180 \\
25,602 \\
27,024\end{array}$ & $\begin{array}{l}60 \\
61 \\
62 \\
63 \\
64 \\
65 \\
66 \\
67 \\
68 \\
69\end{array}$ & $\begin{array}{r}85,340 \\
86,763 \\
\cdot 88,185 \\
89,607 \\
91,030 \\
92,452 \\
93,874 \\
95,297 \\
96,719 \\
98,141\end{array}$ & $\begin{array}{l}110 \\
111 \\
112 \\
113 \\
114 \\
115 \\
116 \\
117 \\
118 \\
119\end{array}$ & $\begin{array}{l}156,457 \\
157,880 \\
159,302 \\
160,724 \\
162,147 \\
163,569 \\
164,991 \\
166,414 \\
167,836 \\
169,238\end{array}$ \\
\hline $\begin{array}{l}15.0 \\
15.5 \\
16.0 \\
16.5 \\
17.0 \\
17.5 \\
18.0 \\
18.5 \\
19.0 \\
19.5\end{array}$ & $\begin{array}{l}33,600 \\
34,720 \\
35,840 \\
36,960 \\
38,080 \\
39,200 \\
40,320 \\
41,440 \\
42,560 \\
43,680\end{array}$ & $\begin{array}{l}40.0 \\
40.5 \\
41.0 \\
41.5 \\
42.0 \\
42.5 \\
43.0 \\
43.5 \\
44.0 \\
44.5\end{array}$ & $\begin{array}{l}89,600 \\
90,720 \\
91,840 \\
92,960 \\
94,080 \\
95,200 \\
96,320 \\
97,440 \\
98,500 \\
99,680\end{array}$ & $\begin{array}{l}80 \\
81 \\
82 \\
83 \\
84 \\
85 \\
86 \\
87 \\
88 \\
89\end{array}$ & $\begin{array}{l}179,200 \\
181,440 \\
183,690 \\
185,920 \\
188,160 \\
190,400 \\
192,640 \\
194,880 \\
197,120 \\
199,360\end{array}$ & $\begin{array}{l}20 \\
21 \\
22 \\
23 \\
24 \\
25 \\
26 \\
27 \\
28 \\
29\end{array}$ & $\begin{array}{l}28,447 \\
29,869 \\
31,291 \\
32,714 \\
34,136 \\
35,558 \\
36,981 \\
38,403 \\
39,826 \\
41,248\end{array}$ & $\begin{array}{l}70 \\
71 \\
72 \\
73 \\
74 \\
75 \\
76 \\
77 \\
78 \\
79\end{array}$ & $\begin{array}{r}99,564 \\
100,986 \\
102,408 \\
103,831 \\
105,253 \\
106,675 \\
108,098 \\
109,520 \\
110,943 \\
112,365\end{array}$ & $\begin{array}{l}120 \\
121 \\
122 \\
123 \\
124 \\
125 \\
126 \\
127 \\
128 \\
129\end{array}$ & $\begin{array}{l}170,681 \\
172,105 \\
173,528 \\
174,940 \\
176,373 \\
177,792 \\
179,215 \\
180,637 \\
182,059 \\
183,482\end{array}$ \\
\hline $\begin{array}{l}20.0 \\
20.5 \\
21.0 \\
21.5 \\
22.0 \\
22.5 \\
23.0 \\
23.5 \\
24.0 \\
24.5\end{array}$ & $\begin{array}{l}44,800 \\
45,920 \\
47,040 \\
48,160 \\
49,280 \\
50,400 \\
51,520 \\
52,640 \\
53,760 \\
54,880\end{array}$ & $\begin{array}{l}45.0 \\
45.5 \\
46.0 \\
46.5 \\
47.0 \\
47.5 \\
48.0 \\
48.5 \\
49.0 \\
49.5\end{array}$ & $\begin{array}{l}100,800 \\
101,920 \\
103,040 \\
104,160 \\
105,280 \\
106,400 \\
107,520 \\
108,640 \\
109,760 \\
110,880\end{array}$ & $\begin{array}{l}90 \\
91 \\
92 \\
93 \\
94 \\
95 \\
96 \\
97 \\
98 \\
99\end{array}$ & $\begin{array}{l}201,600 \\
203,840 \\
206,080 \\
208,320 \\
210,560 \\
212,800 \\
215,040 \\
217,280 \\
219,520 \\
221,760\end{array}$ & $\begin{array}{l}30 \\
31 \\
32 \\
33 \\
34 \\
35 \\
36 \\
37 \\
38 \\
39\end{array}$ & $\begin{array}{l}42,670 \\
44,093 \\
45,515 \\
46,937 \\
48,360 \\
49,782 \\
51,204 \\
52,627 \\
54,049 \\
55,471\end{array}$ & $\begin{array}{l}80 \\
81 \\
82 \\
83 \\
84 \\
85 \\
86 \\
87 \\
88 \\
89\end{array}$ & $\begin{array}{l}113,787 \\
115,210 \\
116,632 \\
118,054 \\
119,477 \\
120,899 \\
122,321 \\
123,744 \\
125,166 \\
126,588\end{array}$ & $\begin{array}{l}130 \\
131 \\
132 \\
133 \\
134 \\
135 \\
136 \\
137 \\
138 \\
139\end{array}$ & $\begin{array}{l}184,904 \\
186,327 \\
187,749 \\
189,171 \\
190,594 \\
192,016 \\
193,438 \\
194,861 \\
196,283 \\
197,705\end{array}$ \\
\hline $\begin{array}{l}25.0 \\
25.5 \\
26.0 \\
26.5 \\
27.0 \\
27.5 \\
28.0 \\
28.5 \\
29.0 \\
29.5\end{array}$ & $\begin{array}{l}56,000 \\
57,120 \\
58,240 \\
59,360 \\
60,480 \\
61,600 \\
62,720 \\
63,840 \\
64,960 \\
66,080\end{array}$ & $\begin{array}{l}50.0 \\
51 \\
52 \\
-53 \\
54 \\
55 \\
56 \\
57 \\
58 \\
59\end{array}$ & $\begin{array}{l}112,000 \\
114,240 \\
116,480 \\
118,720 \\
120,960 \\
123,200 \\
125,440 \\
127,690 \\
129,920 \\
132,160\end{array}$ & $\begin{array}{l}100 \\
101 \\
102 \\
103 \\
104 \\
105 \\
106 \\
107 \\
108 \\
109\end{array}$ & $\begin{array}{l}224,000 \\
226,240 \\
228,480 \\
230,720 \\
232,960 \\
235,200 \\
237,440 \\
239,680 \\
241,920 \\
244,160\end{array}$ & $\begin{array}{l}40 \\
41 \\
42 \\
43 \\
44 \\
45 \\
46 \\
47 \\
48 \\
49\end{array}$ & $\begin{array}{l}56,394 \\
58,316 \\
59,738 \\
61,161 \\
62,583 \\
64,005 \\
65,428 \\
66,850 \\
68,272 \\
69,695\end{array}$ & $\begin{array}{l}90 \\
91 \\
92 \\
93 \\
94 \\
95 \\
96 \\
97 \\
98 \\
99\end{array}$ & $\begin{array}{l}128,011 \\
129,433 \\
120,855 \\
122,278 \\
133,700 \\
135,122 \\
136,545 \\
137,967 \\
139,389 \\
140,912\end{array}$ & $\begin{array}{l}140 \\
141 \\
142 \\
143 \\
144 \\
145 \\
146 \\
147 \\
148 \\
149\end{array}$ & $\begin{array}{l}199,128 \\
200,550 \\
201,972 \\
203,395 \\
204,817 \\
206,239 \\
207,662 \\
209,084 \\
210,506 \\
211,929\end{array}$ \\
\hline $\begin{array}{l}30.0 \\
30.5 \\
31.0 \\
31.5 \\
32.0 \\
32.5 \\
33.0 \\
33.5 \\
34.0 \\
34.5\end{array}$ & $\begin{array}{l}67,200 \\
68,320 \\
69,440 \\
70,560 \\
71,680 \\
72,800 \\
73,920 \\
75,040 \\
76,160 \\
77,280\end{array}$ & $\begin{array}{l}60 \\
61 \\
62 \\
63 \\
64 \\
65 \\
66 \\
67 \\
68 \\
69\end{array}$ & $\begin{array}{l}134,400 \\
136,640 \\
138,880 \\
141,120 \\
143,360 \\
145,600 \\
147,840 \\
150,080 \\
152,320 \\
154,560\end{array}$ & $\begin{array}{l}110 \\
111 \\
112 \\
113 \\
114 \\
115 \\
116 \\
117 \\
118 \\
119\end{array}$ & $\begin{array}{l}246,400 \\
248,640 \\
250,880 \\
253,120 \\
255,360 \\
257,600 \\
259,840 \\
262,080 \\
264,320 \\
266,560\end{array}$ & $\begin{array}{l}50 \\
51 \\
52 \\
53 \\
54 \\
55 \\
56 \\
57 \\
58 \\
59\end{array}$ & $\begin{array}{l}71,117 \\
72,539 \\
73,962 \\
75,384 \\
76,806 \\
78,229 \\
79,651 \\
81,073 \\
82,496 \\
83,918\end{array}$ & $\begin{array}{l}100 \\
101 \\
102 \\
103 \\
104 \\
105 \\
106 \\
107 \\
108 \\
109\end{array}$ & $\begin{array}{l}142,234 \\
143,656 \\
145,079 \\
146,501 \\
147,923 \\
149,346 \\
1507,68 \\
152,190 \\
153,613 \\
155,035\end{array}$ & $\begin{array}{l}150 \\
151 \\
152 \\
153 \\
154 \\
155 \\
156 \\
157 \\
158 \\
159\end{array}$ & $\begin{array}{l}213,351 \\
214,773 \\
216,196 \\
217,618 \\
219,040 \\
220,463 \\
221,885 \\
223,307 \\
224,730 \\
226,152\end{array}$ \\
\hline
\end{tabular}

\section{諸 單 位 換 算 表}

$\mathrm{Km} . \times .621=\mathrm{mi}$.

Km. $\div-1.609=\mathrm{mi}$.

m. $\times 39.37=$ in.

$\mathrm{m} . \times 3.281=\mathrm{ft}$.

m. $\times 1.094=y d$.

cm. $\times .3937=$ in. cm. $\div 2.54=$ in

m.m. $\times .03937=$ in.

m.m. $\div-25.4=$ in.

sq. Km. $\times 247.1=$ A.

sq. $\mathrm{m} \times 10.764=\mathrm{sq} . \mathrm{ft}$.

sq.cm $\times .155=$ sq.in. sq.cm. $\div 6.451=$ sq.in. sq.mm. $\times .0015=$ sq.in. sq.mm. $\div 645.1=$ sq.in. cu.m. $\times 35.315=$ cu.ft. cu.m. $\times 1.308=$ cu.yd. cu.m. $\div 264.2=$ gal.(U.S.) cu.cm. $-16.383=$ cu.in.

1. $\times 61.022=$ cu.in.

$1 . \div 2642=$ gal. (U.S.)

$1 . \times 3.78=$ gal.(U.S.)

1.- $-28.16=$ cu.ft. 
溫 度 換 算 㤗

\begin{tabular}{|c|c|c|c|c|c|c|c|c|c|c|}
\hline $\mathrm{C}^{\circ}$ & 0 & 10 & 20 & 30 & 40 & 50 & 60 & 70 & 80 & 90 \\
\hline-200 & $\begin{array}{c}\mathrm{F} \\
-328\end{array}$ & $\begin{array}{c}\mathrm{F} \\
-346\end{array}$ & $\begin{array}{c}\mathrm{F} \\
-364\end{array}$ & $\begin{array}{c}\mathrm{F} \\
-382\end{array}$ & $\begin{array}{c}F \\
-400\end{array}$ & $\begin{array}{c}F \\
-418\end{array}$ & $\begin{array}{c}F \\
-436\end{array}$ & $\begin{array}{c}F \\
-454\end{array}$ & $\bar{F}$ & $\mathbf{F}$ \\
\hline-100 & -148 & $\begin{array}{l}-166 \\
\end{array}$ & $\begin{array}{l}-504 \\
-184\end{array}$ & $\begin{array}{l}-302 \\
-202\end{array}$ & -220 & -238 & -256 & -274 & -292 & $\begin{array}{l}-310 \\
-310\end{array}$ \\
\hline-0 & +32 & +14 & -4 & -22 & -40 & -58 & -76 & -94 & & -130 \\
\hline & 32 & 50 & $6 \overline{8}$ & 86 & 104 & 122 & 140 & 158 & 176 & 194 \\
\hline 100 & 212 & 230 & 248 & 266 & 284 & 302 & 320 & 338 & 356 & 374 \\
\hline 200 & 392 & 410 & 428 & 446 & 464 & 482 & 500 & 518 & 536 & 554 \\
\hline 300 & 572 & 590 & 608 & 626 & 644 & 662 & 680 & 698 & 716 & 734 \\
\hline 400 & 752 & 770 & 788 & 806 & 824 & 842 & 860 & 878 & 896 & 914 \\
\hline 500 & 932 & 950 & 968 & 986 & 1004 & 1022 & 1040 & 1058 & 1076 & 1094 \\
\hline $60^{0}$ & 1112 & 1130 & 1148 & 1166 & 1184 & 1202 & 1220 & 1238 & 1256 & 1274 \\
\hline 700 & 1292 & 1310 & 1328 & 1346 & 1364 & 1382 & 1400 & 1418 & 1436 & 1454 \\
\hline 800 & 1472 & 1490 & 1508 & 1526 & 1544 & 1562 & 1580 & 1598 & 1616 & 1634 \\
\hline 900 & 1652 & 1970 & 1698 & 1706 & 1724 & 1742 & 1760 & 1778 & 1796 & 1814 \\
\hline 1000 & 1832 & 1850 & 1868 & 1886 & 1904 & 1922 & 1940 & 1958 & 1976 & 1994 \\
\hline 1100 & 2012 & 2030 & 2048 & 2066 & 2084 & 2102 & 2120 & 2138 & 2156 & 2174 \\
\hline 1200 & 2192 & 2210 & 2228 & 2246 & 2264 & 2292 & 2300 & 2318 & 2336 & 2354 \\
\hline 1300 & 2372 & 2390 & 2408 & 2426 & 2444 & 2462 & 2480 & 2498 & 2516 & 2534 \\
\hline 1400 & 2552 & 2570 & 2588 & 2606 & 2624 & 2642 & 2660 & 2678 & 2696 & 2714 \\
\hline 1500 & 2732 & 2750 & 2768 & 2786 & 2804 & 2822 & 2840 & 2858 & 2876 & 2894 \\
\hline 1600 & 2912 & 2930 & 2948 & 2966 & 2984 & 3022 & 3020 & 3038 & 3056 & 3074 \\
\hline 1700 & 3092 & 3110 & 3128 & 3146 & 3164 & 3192 & 3200 & 3218 & 3236 & 3254 \\
\hline 1800 & 3272 & 3290 & 3308 & 3326 & 3344 & 3362 & 3380 & 3398 & 3416 & 3434 \\
\hline 1900 & 3452 & 3570 & 3408 & 3506 & 3534 & 3542 & 3560 & 3578 & 3596 & 3614 \\
\hline 2000 & 3632 & 3650 & 3668 & 3686 & 3704 & 3722 & 3740 & 3758 & 3776 & 3774 \\
\hline 2100 & 3812 & 3830 & 3848 & 3866 & 3894 & $3 \overline{902}$ & 3920 & 3938 & 3936 & 3974 \\
\hline 2200 & 3992 & 4010 & 4028 & 4046 & 4064 & 4082 & 4100 & 4118 & 4136 & 4154 \\
\hline 2300 & 4172 & 4190 & 4208 & 4226 & 4244 & 4262 & 4280 & 4298 & 4316 & 4334 \\
\hline 2400 & 4352 & 4370 & 4388 & 4406 & 4424 & 4442 & 4460 & 4478 & 4496 & 4514 \\
\hline $\begin{array}{l}2500 \\
2600\end{array}$ & 4532 & 4550 & 4568 & 4586 & 4604 & 4622 & 4640 & 4658 & 4676 & 4694 \\
\hline 2600 & 4712 & 4730 & 4738 & 4766 & 4784 & 4802 & 4820 & 4838 & 4856 & 4874 \\
\hline 2700 & 4892 & 4910 & 4928 & 4946 & 4964 & 4982 & 5000 & 5018 & 5036 & 5054 \\
\hline 2800 & 5072 & 5090 & 5108 & 5126 & 5144 & 5162 & 5182 & 5198 & 5216 & 5234 \\
\hline 2900 & 5252 & 5270 & 5288 & 5306 & 5324 & 5342 & 5360 & 3578 & 5396 & 5414 \\
\hline 3000 & 5432 & 5450 & 5468 & 5486 & 5504 & 5522 & 5540 & 5558 & 5576 & 5594 \\
\hline 3100 & 5612 & 5630 & 5648 & 5666 & 5684 & 5702 & 5720 & 5738 & 5756 & 5774 \\
\hline 3200 & 5792 & 5810 & 5828 & 5846 & 5864 & 5882 & 5900 & 5918 & 5936 & 5954 \\
\hline 3300 & 5972 & 5990 & 6008 & 6026 & 6044 & 6062 & 6080 & 6098 & 6116 & 6134 \\
\hline $\mathbf{3 4 0 0}$ & 6152 & 6170 & 6188 & 6206 & 6224 & 6242 & 6260 & 6278 & 6296 & 6314 \\
\hline 3500 & 6332 & 6350 & 6368 & 6386 & 6404 & 6422 & 6440 & 6458 & 6476 & 6494 \\
\hline 3600 & 6512 & 6530 & 6548 & 6566 & 6584 & 6602 & 6620 & 6638 & 6656 & 6674 \\
\hline 3700 & 6692 & 6710 & 6728 & 6746 & 6764 & 6782 & 6800 & 6818 & 6836 & 6854 \\
\hline 3800 & 6872 & 6890 & 6908 & 6926 & 694 & 6962 & 6980 & 6998 & 7016 & 7034 \\
\hline 3900 & 7052 & 7070 & 7088 & 7106 & 7124 & 7142 & 7160 & 7178 & 7196 & 7214 \\
\hline $\mathrm{C}^{\circ}$ & 0 & 10 & 20 & 30 & 40 & 50 & 60 & 70 & 80 & 90 \\
\hline
\end{tabular}

插間值 $\mathrm{C}^{\circ} \quad \mathrm{F}^{\circ}$ $1 \ldots \ldots . . .1 .8$ $2 \ldots \ldots \ldots 3.6$ $3 \cdots \cdots \cdot 5.4$ $4 \ldots \ldots \ldots .7 .2$ $5 \ldots \ldots \ldots .9 .2$ $6 \cdot \ldots \ldots \ldots 10.8$

$7 \ldots \ldots \ldots 12.6$

8.........14.4 $9 \ldots \ldots \ldots . .16 .2$ $10 \cdots \cdots \cdots .18 .0$

$\mathrm{F}^{\circ} \quad \mathrm{C}^{\mathrm{O}}$ $1 \ldots \ldots \ldots 0.56$ $2 \ldots \ldots \ldots 1.11$ $3 \ldots \ldots \ldots 1.67$

$4 \ldots \ldots \ldots .2 .22$

$5 \ldots \ldots \ldots 2.78$

6 ........ 3.33

$7 \ldots \ldots \ldots .3 .89$

$8 \ldots \ldots \ldots .4 .44$

$9 . \ldots \ldots . . .5 .00$

$10 \ldots \ldots \ldots .56$

$11 \ldots \ldots \ldots 6.11$

$12 \cdots \ldots \cdots 6.67$

$13 \ldots \ldots \ldots, 7.22$

$14 \ldots \ldots \ldots 7.78$

$15 \ldots \ldots \ldots . .33$

$16 \ldots \ldots . .8 .89$

$17 \ldots \ldots \ldots 9.44$

$18 \cdot \cdots \cdots \cdots \cdot 10.00$

\section{諸 單 位 換 算 表}

g. $\times 15.432=\mathrm{gr}$.

g. $\times 981=$ dynes

g. $\div \cdot-28.35=0 z$. (avoir.)

grams per sq. $\mathrm{cm} . \times 14,22=1 \mathrm{~b}$.per sq. in.

$\mathrm{kg} \cdot \times 2.205=1 \mathrm{~b}$.

$\mathrm{kg} . \times 35=30 \mathrm{z}$. (avoir.)

kg. $\times 1,102.3=$ tons $(20001 b$.)

kg.persq.cm. $\times 14.233=1$ b.per sq. in.

kg.m. $\times 7.233=\mathrm{ft} .1 \mathrm{~b}$

kilowatts $(\mathrm{k} . \mathrm{w}) \times 1.34=$. H.P. watts $\div 746=$ H.P.

watts $\times .7373=$ ft.1b.per sec.

Joules $\times .7373=\mathrm{ft}$. $1 \mathrm{~b}$.

Calorie (kilogram-degr.C.) $\times 3.968=$ B.T.U.

Cal orie (kilogram-degr.C.) $\div .252=$ B.T.U.

Joules $\times .24=$ gram-caloris

gram-calories $\times 4.19=$ Joules

gravity (paris) $=981 \mathrm{~cm}$.per $\mathrm{sec}$

per sec.

(Degrees Centigrade $\times 1.8)+32^{\circ}=$ degrees $\mathrm{F}$. 